\title{
Solubility of Chlordiazepoxide, Diazepam, and Lorazepam in Ethanol + Water Mixtures at 303.2 K
}

\author{
Abolghasem Jouyban, ${ }^{*}, \#$ Javad Shokri, ${ }^{\dagger}$ Mohammad Barzegar-Jalali, ${ }^{\dagger}$ Davoud Hassanzadeh, ${ }^{\dagger}$ William E. Acree, Jr., ${ }^{\S}$

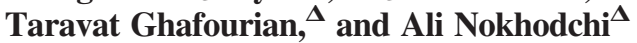

Faculty of Pharmacy and Drug Applied Research Center, Biotechnology Research Center, Research Center for Pharmaceutical Nanotechnology, Tabriz University (Medical Sciences), Tabriz 51664, Iran, Department of Chemistry, University of North Texas, Denton, Texas 76203-5070, and Medway School of Pharmacy, Universities of Kent and Greenwich,

Kent ME4 4TB, United Kingdom

\begin{abstract}
Experimental solubilities of three benzodiazepines (chlordiazepoxide, diazepam, and lorazepam) in ethanol + water mixtures at $303.2 \mathrm{~K}$ are reported. The solubility of drugs was increased with the addition of ethanol and reached the maximum value of a volume fraction of $90 \%$ of ethanol. The Jouyban-Acree model was used to fit the experimental data, and the solubilities were reproduced using previously trained version of the Jouyban-Acree model and the solubility data in monosolvents in which the overall mean relative deviations (OMRDs) of the models were 8.6\%, $21.9 \%$ and $19.3 \%$, respectively, for the fitted model, the trained version for ethanol + water mixtures and generally trained version for various organic solvents + water mixtures.
\end{abstract}

\section{Introduction}

Ethanol is a common and safe cosolvent to be used in pharmaceutical liquid formulations. Its solubilization power is reasonably high and usually used in the liquid formulations at concentrations lower than $50 \%$. In addition to solubility enhancement of ethanol, it can affect a drug's absorption, distribution, metabolism, and excretion. Absorption of a drug can be increased by increasing gastrointestinal (GI) blood flow when higher concentration of ethanol exists in the GI fluid. A higher concentration of ethanol may also cause a delay in drug absorption by the induced pyloric spasm. ${ }^{1}$ It has also been shown that drugs could affect the bioavailability of ethanol. ${ }^{2}$

The solubility of drugs in ethanol + water mixtures is essential preformulation information. The data could be used in recrystallization and also in formulation processes. The concentration of ethanol in pharmaceutical preparations should be kept as low as possible. The method used to optimize the solvent composition of the mixtures for dissolving a desired amount of a drug in a given volume of the solution is the trial and error approach, which is timeconsuming and expensive. Moreover, in the early stages of drug discovery processes, the scarcity of the available amount of drug/drug candidate is another limiting factor. To address this issue, a number of mathematical models have been presented for predicting the solubility of drugs in watercosolvent mixtures. These models and their advantages and limitations were recently reviewed. ${ }^{3}$

\footnotetext{
* To whom correspondence should be addressed. E-mail: ajouyban@ hotmail.com. Fax: +98 4113363231.

\# Faculty of Pharmacy and Drug Applied Research Center, Tabriz University (Medical Sciences).

Biotechnology Research Center, Tabriz University (Medical Sciences).

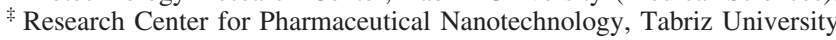
(Medical Sciences).

$\$$ University of North Texas.

${ }^{\Delta}$ Universities of Kent and Greenwich.
}

Table 1. Abraham Solute Parameters of the Drugs Computed by PharmaAlgorithm 6

\begin{tabular}{lccccc}
\hline \multicolumn{1}{c}{ drug } & $E$ & $S$ & $A$ & $B$ & $V$ \\
\hline chlordiazepoxide & 1.95 & 1.65 & 0.13 & 0.94 & 2.17 \\
diazepam & 2.11 & 1.72 & 0.00 & 1.04 & 2.00 \\
lorazepam & 2.37 & 1.83 & 0.64 & 1.29 & 2.11
\end{tabular}

Table 2. Abraham Solvent Coefficients Employed in This Work ${ }^{7}$

\begin{tabular}{lrrrrrr}
\hline solvent & $c$ & $e$ & \multicolumn{1}{c}{$s$} & \multicolumn{1}{c}{$a$} & \multicolumn{1}{c}{$b$} & \multicolumn{1}{c}{$v$} \\
\hline ethanol & 0.208 & 0.409 & -0.959 & 0.186 & -3.645 & 3.928 \\
water & -0.994 & 0.577 & 2.549 & 3.813 & 4.841 & -0.869
\end{tabular}

Of the numerous models developed in recent years, the Jouyban-Acree model is perhaps one of the more versatile models. The model provides very accurate mathematical descriptions for how the solute solubility varies with both temperature and solvent composition. The model is

$$
\begin{aligned}
\log x_{m, T}^{\mathrm{Sat}}=\varphi_{1} \log x_{1, T}^{\mathrm{Sat}}+\varphi_{2} \log x_{2, T}^{\mathrm{Sat}}+ & \\
& \frac{\varphi_{1} \varphi_{2}}{T} \sum_{i=0}^{2} J_{i}\left(\varphi_{1}-\varphi_{2}\right)^{i}
\end{aligned}
$$

where $x_{m, T}^{\text {Sat }}$ is the solute (mole fraction) solubility in the binary solvent mixtures at temperature $T / \mathrm{K}, \varphi_{1}$, and $\varphi_{2}$ are the volume fractions of the solvents 1 (ethanol) and 2 (water) in the absence of the solute, $x_{1, T}^{\text {Sat }}$ and $x_{2, T}^{\text {Sat }}$ denote the mole fraction solubility of the solute in the neat solvents 1 and 2 , respectively, and $J_{i}$ are the constants of the model computed by a regression analysis. ${ }^{3}$ The existence of these model constants, which require a number of solubility data in water-cosolvent mixtures for training process, is a limitation for the model when the solubility predictions are the goal of the computations in early drug discovery studies. This limitation could be resolved using a trained version of the model for a given water-cosolvent mixture. The trained 
Table 3. Details of Calibration Curves of Drugs

\begin{tabular}{|c|c|c|c|c|c|}
\hline & $\lambda$ & $\varepsilon$ & $c$ & & \\
\hline drug & $\mathrm{nm}$ & $\mathrm{L} \cdot \mathrm{mol}^{-1} \cdot \mathrm{cm}^{-1}$ & $\mathrm{~mol} \cdot \mathrm{L}^{-1}$ & correl coeff & calibration curve ( $A$ : absorbance) \\
\hline chlordiazepoxide & 234 & 30167 to 31568 & $2.25 \times 10^{-6}$ to $3.34 \times 10^{-5}$ & 0.999 & $c=3.326 \times 10^{-5} A-1.125 \times 10^{-7}$ \\
\hline diazepam & 231 & 33234 to 38033 & $2.37 \times 10^{-6}$ to $3.51 \times 10^{-5}$ & 0.999 & $c=3.041 \times 10^{-5} A-3.710 \times 10^{-7}$ \\
\hline lorazepam & 231 & 37980 to 38924 & $2.10 \times 10^{-5}$ to $3.11 \times 10^{-5}$ & 0.999 & $c=2.638 \times 10^{-5} A-5.615 \times 10^{-8}$ \\
\hline
\end{tabular}

Table 4. Experimental Mole Fraction Solubilities of Chlordiazepoxide, Diazepam, and Lorazepam in Different Volume Fractions of Ethanol $\left(\varphi_{1}\right)$ in Ethanol (1) + Water (2) Mixtures at 303.2 K and Density $\rho$ of the Saturated Solutions

\begin{tabular}{|c|c|c|c|c|c|c|}
\hline \multirow[b]{2}{*}{$\varphi_{1}$} & \multicolumn{3}{|c|}{$x_{m, T}^{\mathrm{Sat}}$} & \multicolumn{3}{|c|}{$\rho / \mathrm{g} \cdot \mathrm{cm}^{-3}$} \\
\hline & chlordiazepoxide & diazepam & lorazepam & chlordiazepoxide & diazepam & lorazepam \\
\hline 0.000 & 0.000006 & 0.000003 & 0.000003 & 0.998 & 0.995 & 0.991 \\
\hline 0.100 & 0.000012 & 0.000008 & 0.000007 & 0.995 & 0.992 & 0.986 \\
\hline 0.200 & 0.000021 & 0.000020 & 0.000017 & 0.970 & 0.971 & 0.977 \\
\hline 0.300 & 0.000066 & 0.000074 & 0.000066 & 0.953 & 0.957 & 0.961 \\
\hline 0.400 & 0.000270 & 0.000287 & 0.000336 & 0.931 & 0.952 & 0.952 \\
\hline 0.500 & 0.000769 & 0.000976 & 0.000449 & 0.927 & 0.933 & 0.932 \\
\hline 0.600 & 0.001450 & 0.002190 & 0.000959 & 0.892 & 0.912 & 0.909 \\
\hline 0.700 & 0.002940 & 0.004490 & 0.001730 & 0.872 & 0.891 & 0.887 \\
\hline 0.800 & 0.004430 & 0.006790 & 0.002530 & 0.864 & 0.853 & 0.863 \\
\hline 0.900 & 0.004990 & 0.008580 & 0.002690 & 0.818 & 0.831 & 0.833 \\
\hline 1.000 & 0.003350 & 0.007540 & 0.001960 & 0.796 & 0.784 & 0.797 \\
\hline
\end{tabular}

version of the Jouyban-Acree model for prediction of drugs solubility in ethanol + water mixtures at temperature $T$ is ${ }^{4}$

$$
\begin{gathered}
\log x_{m, T}^{\text {Sat }}=\varphi_{1} \log x_{1, T}^{\text {Sat }}+\varphi_{2} \log x_{2, T}^{\text {Sat }}+\frac{724.21 \varphi_{1} \varphi_{2}}{T}+ \\
\frac{458.17 \varphi_{1} \varphi_{2}\left(\varphi_{1}-\varphi_{2}\right)}{T}+\frac{194.21 \varphi_{1} \varphi_{2}\left(\varphi_{1}-\varphi_{2}\right)^{2}}{T}
\end{gathered}
$$

Equation 2 is only applicable for solubility prediction of drugs in ethanol + water mixtures and the effect of drug structures on the solubility was ignored. To provide a general model and also to consider the chemical structure of the drugs, the QSPR models for computing the constants of the Jouyban-Acree model ( $J_{i}$ terms) using Abraham solvation parameters (for both solvent and drugs) were reported as ${ }^{5}$

$$
\begin{aligned}
& J_{0}=2113.119-1093.783\left(c_{1}-c_{2}\right)^{2}+3380.661 E\left(e_{1}-e_{2}\right)^{2}- \\
& 13.865 S\left(s_{1}-s_{2}\right)^{2}-4.921 A\left(a_{1}-a_{2}\right)^{2}-5.659 B\left(b_{1}-b_{2}\right)^{2}+ \\
& 15.250 V\left(v_{1}-v_{2}\right)^{2}(3) \\
& J_{1}=-2001.561+1142.780\left(c_{1}-c_{2}\right)^{2}-2735.160 E\left(e_{1}-\right. \\
& \left.e_{2}\right)^{2}- \\
& 38.541 S\left(s_{1}-s_{2}\right)^{2}+13.176 A\left(a_{1}-a_{2}\right)^{2}+0.811 B\left(b_{1}-b_{2}\right)^{2}+ \\
& 38.508 V\left(v_{1}-v_{2}\right)^{2}(4) \\
& J_{2}=1474.963-1507.479\left(c_{1}-c_{2}\right)^{2}+4421.302 E\left(e_{1}-e_{2}\right)^{2}+ \\
& 17.981 S\left(s_{1}-s_{2}\right)^{2}-21.196 A\left(a_{1}-a_{2}\right)^{2}+6.595 B\left(b_{1}-b_{2}\right)^{2}- \\
& 13.386 V\left(v_{1}-v_{2}\right)^{2}(5)
\end{aligned}
$$

where $c, e, s, a, b$, and $v$ are the solvent coefficients, subscripts 1 and 2 denote cosolvent and water, respectively, $E$ is the excess molar refraction solute descriptor, $S$ is the dipolarity/polarizability of the solute, $A$ denotes the solute's hydrogen-bond acidity, $B$ stands for the solute's hydrogen-bond basicity, and $V$ is the McGowan volume of the solute in unit of $0.01\left(\mathrm{~cm}^{3} \cdot \mathrm{mol}^{-1}\right)$. The solvent coefficients (c,e,s,a,b, and $\left.v\right)$ are obtained by regression analysis of experimental data for a specific process (partitioning between two solvents) and represent differences in the solvent phase properties. The numerical values of Abraham solute parameters of the drugs computed by PharmaAlgorithm ${ }^{6}$ and the Abraham solvent coefficients ${ }^{7}$ employed in this work are listed in Tables 1 and 2, respectively.
These terms represent various chemical interactions in the solution. $^{5}$

Experimental solubility of diazepam in ethanol + water mixtures at $298.15 \mathrm{~K}$ was reported in a previous work. ${ }^{8} \mathrm{In}$ this work, the experimental solubility of chlordiazepoxide, diazepam, and lorazepam in ethanol + water mixtures at $303.2 \mathrm{~K}$ are reported. There is no published solubility data for these drugs in ethanol + water mixtures at $303.2 \mathrm{~K}$. In addition, the applicability of the Jouyban-Acree model to the measured drug solubility data and the prediction capability of above-mentioned trained models for predicting the solubility of drugs in ethanol + water mixtures were investigated.

\section{Experimental Method}

Materials. Chlordiazepoxide, diazepam, and lorazepam were purchased from Loghman pharmaceutical company (Iran). Ethanol (99.9\%) was purchased from Merck (Germany), methanol (99.8\%) was obtained from Caledon (Canada), and double distilled water was used for preparation of the solutions.

Apparatus and Procedures. The binary solvent mixtures were prepared by mixing the appropriate volumes of the solvents with the accuracy of 0.001 volume fraction. The solubility of benzodiazepines in ethanol + water mixtures was determined

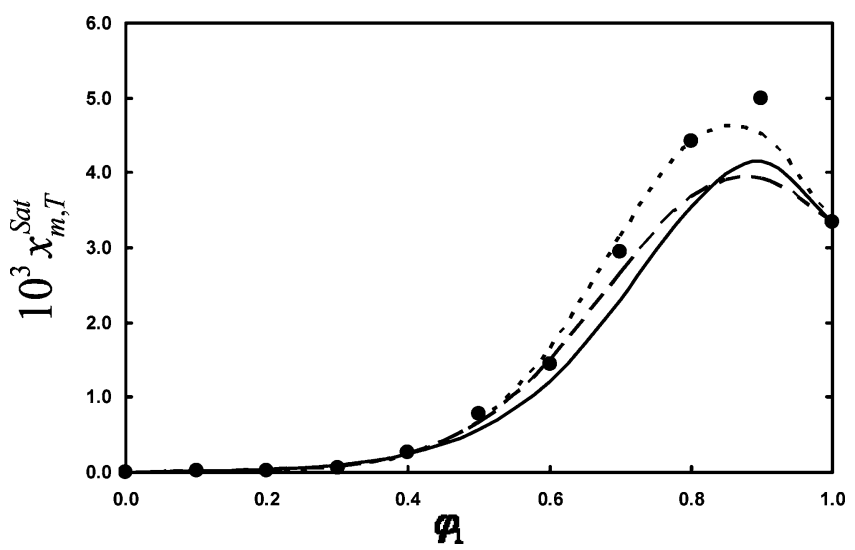

Figure 1. Mole fraction solubility of chlordiazepoxide $\left(x_{m, T}^{\mathrm{Sat}}\right)$ at various volume fractions of ethanol $\left(\varphi_{1}\right)$ in binary solvent mixtures: $(\bullet)$ experimental; the computed solubilities using (---) method I, (-) method II, and (-- ) method III. 


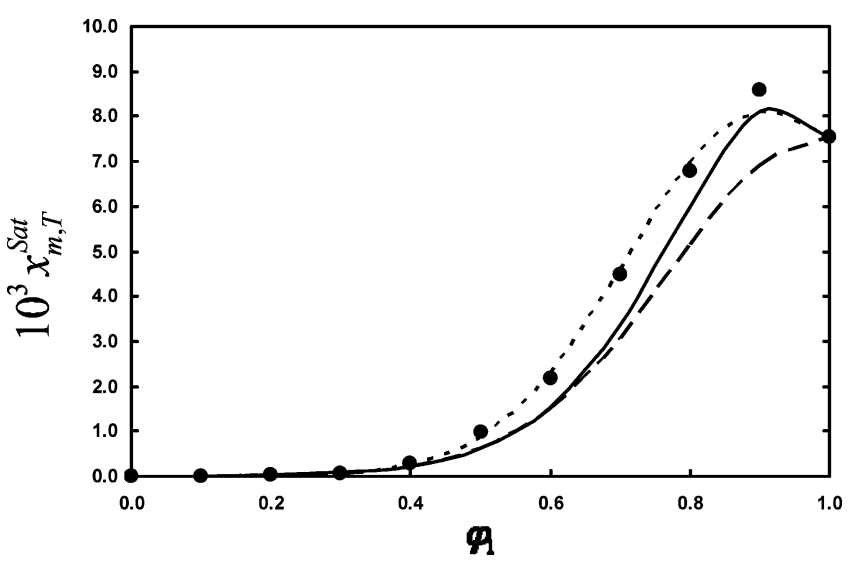

Figure 2. Mole fraction solubility of diazepam $\left(x_{m, T}^{\mathrm{Sat}}\right)$ at various volume fractions of ethanol $\left(\varphi_{1}\right)$ in binary solvent mixtures: $(\bullet)$ experimental; the computed solubilities using (--) method I, (-) method II, and (---) method III.

by equilibrating excess amounts of the solids at $303.2 \mathrm{~K}$ using a shaker (Behdad, Tehran, Iran) placed in an incubator equipped with a temperature-controlling system maintained constant within $\pm 0.2 \mathrm{~K}$. After a sufficient length of time $(>24 \mathrm{~h})$, the saturated solutions of the drugs were filtered using hydrophilic Durapore filters $(0.45 \mu \mathrm{m}$, Milipore, Ireland), diluted with methanol, and then assayed at $234 \mathrm{~nm}, 231 \mathrm{~nm}$, and $231 \mathrm{~nm}$, respectively for chlordiazepoxide, diazepam, and lorazepam using a UV-vis spectrophotometer (Beckman DU-650, Fullerton). The preliminary investigations showed that the filter did not absorb the solutes through the filtration process. Concentrations of the diluted solutions were determined from the calibration curves. Details of calibration curves are shown in Table 3. Each experimental data point represents the average of at least three repetitive experiments with the measured mole fraction solubilities being reproducible on a relative basis within $\pm 4.2 \%$. The standard relative deviations of mole fraction solubilities ranged from $1.0 \%$ to $6.6 \%$. The densities of the saturated solutions were determined using a $5 \mathrm{~mL}$ pycnometer with the uncertainty of $\left(\sigma_{n-1}=0.003\right.$ to $\left.\sigma_{n-1}=0.018\right) \mathrm{g} \cdot \mathrm{cm}^{-3}$.

Computational Methods. In the numerical analysis of method I, eq 1 was fitted to the experimental solubility data of each drug and the back-calculated solubilities were used to calculate the accuracy of the fit. In method II, the solubilities of three drugs were predicted using eq 2 employing the experimental solubilities of drugs in ethanol and water. In method III, solubilities of these drugs were predicted using the model constants of eq 1 predicted by eqs 3 to 5 and their solubility in neat water and ethanol at $303.2 \mathrm{~K}$. The mean relative deviation (MRD) was used to check the accuracy of the predictions using

$$
\mathrm{MRD}=\frac{\sum\left\{\frac{\left|\left(x_{m}^{\text {Sat }}\right)_{\text {pred }}-\left(x_{m}^{\text {Sat }}\right)\right|}{\left(x_{m}^{\text {Sat }}\right)}\right\}}{N}
$$

where $N$ is the number of data points in each set. The goodness

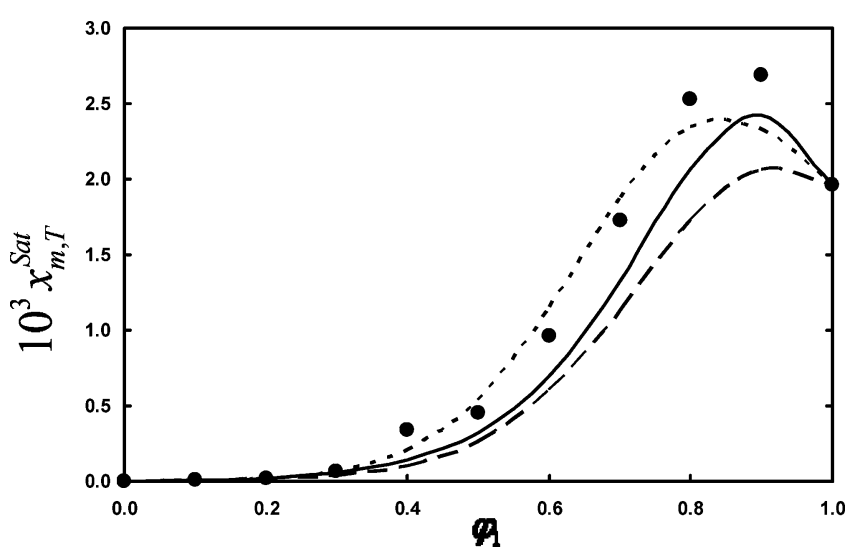

Figure 3. Mole fraction solubility of lorazepam $\left(x_{m, T}^{\mathrm{Sat}}\right)$ at various volume fractions of ethanol $\left(\varphi_{1}\right)$ in binary solvent mixtures: $(\bullet)$ experimental; the computed solubilities using (---) method I, (-) method II, and (---) method III.

of fit to each method was also shown by plotting the predicted and experimental solubilities of the drugs against the volume fraction of ethanol.

\section{Results and Discussion}

Table 4 lists the experimental solubilities of chlordiazepoxide, diazepam, and lorazepam in ethanol + water mixtures at 303.2 $\mathrm{K}$. There are agreements between aqueous solubility data of diazepam at $303.2 \mathrm{~K}$ from this work $\left(0.00018 \mathrm{~mol} \cdot \mathrm{L}^{-1}\right)$, a published solubility data of diazepam in water at $303.15 \mathrm{~K}$ $\left(0.00014 \mathrm{~mol} \cdot \mathrm{L}^{-1}\right),{ }^{9}$ and a recently reported solubility data of diazepam in water at $298.15 \mathrm{~K}\left(0.00015 \mathrm{~mol} \cdot \mathrm{L}^{-1}\right)^{8}$ given that solubilities of insoluble crystalline solutes are often difficult to measure. Comparing these three values concerning the temperature, reveal that $0.00014 \mathrm{~mol} \cdot \mathrm{L}^{-1}$ is an underestimated datum. There is also a parallel shift in solubility data of diazepam at various solvent compositions of ethanol + water mixtures at $298.15^{8}$ and the corresponding data at $303.2 \mathrm{~K}$ from this work. The solubility of drugs increased with the addition of ethanol, reached the maximum values at $\varphi_{1}=0.9$ and then decreased again in neat ethanol.

The solubility of the benzodiazepines was predicted using numerical methods I to III. The experimental and predicted solubilities of the drugs versus the volume fraction of ethanol in the binary mixtures were plotted in Figures 1, 2, and 3. As shown in the figures, the Jouyban-Acree model fits very well to the experimental solubility data of drugs at all composition ranges of ethanol. This finding is also supported by small MRD values of the back-calculated and experimental solubility data. The main limitation of eq 1 is that it should be trained for each drug employing a minimum number of experimental data in binary solvents; however, when the constants for each system were calculated, the model could be used to predict the solubility at other solvent compositions ${ }^{10}$ or other temperatures ${ }^{11}$ and the expected prediction MRD is less than $16 \% .^{10,11}$ As further evidence for this finding, the solubility of diazepam at 298.15

Table 5. Numerical Values of Adjusted Parameters of Eq 1 for Each Solute and the Mean Relative Deviation (MRD) for the Predicted Solubilities of Drugs in Ethanol (1) + Water (2) Mixtures Using Various Numerical Analyses and Their Overall Values

\begin{tabular}{|c|c|c|c|c|c|c|}
\hline \multirow[b]{2}{*}{ drug } & \multirow[b]{2}{*}{$J_{0}$} & \multirow[b]{2}{*}{$J_{1}$} & \multirow[b]{2}{*}{$J_{2}$} & \multicolumn{3}{|c|}{$100 \cdot \mathrm{MRD}$} \\
\hline & & & & method I & method II & method III \\
\hline chlordiazepoxide & 815.761 & 973.330 & -367.813 & 9.1 & 26.1 & 13.0 \\
\hline diazepam & 908.489 & 773.923 & -453.683 & 4.5 & 18.5 & 17.1 \\
\hline \multirow[t]{2}{*}{ lorazepam } & 992.663 & 621.135 & -479.387 & 12.1 & 21.1 & 27.8 \\
\hline & & & overall: & 8.6 & 21.9 & 19.3 \\
\hline
\end{tabular}


$\mathrm{K}$ reported in an earlier report ${ }^{8}$ was predicted using the model constants of the diazepam in ethanol + water system at 303.2 $\mathrm{K}$ (reported in Table 5 of this work) and the obtained prediction MRD is $15.0 \%$.

The predictive version of the model, i.e., eq 2 , predicts the solubility values with reasonable MRD values. The predicted solubilities were compared with the corresponding experimental data, and MRD values were computed and listed in Table 5. The prediction procedure using eq 2 is straightforward and could be preferred in solubility predictions in ethanol + water mixtures at various temperatures. However, it is only applicable for ethanol + water mixtures. As noticed above, the $J_{i}$ terms of the Jouyban-Acree model could be predicted using eqs 3 to 5 (requiring Abraham solute parameters and solvent coefficients), and the produced prediction errors are comparable with those of eq 2. Although the computations of method III require more efforts, it could be used for the cosolvents with known Abraham solvent coefficients. Generally the overall MRDs observed in these predictions show that the Jouyban-Acree model is robust and could be used for prediction purposes with a relative uncertainty of less than $22 \%$.

\section{Literature Cited}

(1) Linnoila, M.; Mattila, M. J.; Kitchell, B. S. Drugs Interaction with Alcohols. Drugs 1979, 18, 299-311.

(2) Brown, A. S. J. M.; Fiaterone, J. R.; Day, C. P.; Bennett, M. K.; Kelly, P. J.; James, O. F. Ranitidine increases the Bioavailability of
Postprandial Ethanol by the Reduction of First Pass Metabolism. Gut 1995, 37, 413-417.

(3) Jouyban, A. Review of the Cosolvency Models for Predicting Solubility of Drugs in Water-Cosolvent Mixtures. J. Pharm. Pharm. Sci. 2008, 11, 32-58.

(4) Jouyban, A.; Acree, W. E., Jr. In Silico Prediction of Drug Solubility in Water - Ethanol Mixtures Using Jouyban-Acree Model. J. Pharm. Pharm. Sci. 2006, 9, 262-269.

(5) Jouyban, A.; Soltanpour, S.; Soltani, S.; Chan, H. K.; Acree, W. E., Jr. Solubility Prediction of Drugs in Water-Cosolvent Mixtures Using Abraham Solvation Parameters. J. Pharm. Pharm. Sci. 2007, 10, 294308.

(6) ADME Boxes, Version 4.0; Pharma Algorithms: Toronto 2008.

(7) Stovall, D. M.; Acree, W. E., Jr.; Abraham, M. H. Solubility of 9-Fluorenone, Thianthrene and Xanthene in Organic Solvents. Fluid Phase Equilib. 2005, 232, 113-121.

(8) Shayanfar, A.; Fakhree, M. A. A.; Acree, W. E., Jr.; Jouyban, A. Solubility of Lamotrigine, Diazepam, and Clonazepam in Ethanol + Water Mixtures at 298.15 K. J. Chem. Eng. Data 2009, 54, 11071109.

(9) Rosoff, M.; Serajuddin, A. T. M. Solubilization of Diazepam in Bile Salts and Sodium Cholate-Lecitin-Water Phases. Int. J. Pharm. 1980, 6, 137-146.

(10) Jouyban-Gharamaleki, A.; York, P.; Hanna, M.; Clark, B. J. Solubility Prediction of Salmeterol Xinafoate in Water-Dioxane Mixtures. Int. J. Pharm. 2001, 216, 33-41.

(11) Jouyban, A.; Acree, W. E., Jr. Comments on "Solubility of Ethyl Maltol in Aqueous Ethanol Mixtures". J. Chem. Eng. Data 2009, 54, 11681170 .

Received for review February 21, 2009. Accepted March 28, 2009.

JE900200K 